\title{
USOS DE LA PALABRA "SOSTENIBILIDAD" EN EL DERECHO \\ DEL TRABAJO: ¿RENOVACIÓN, CIRCULARIDAD O REITERACIÓN?
}

Autor convidado

Dr. Hugo Barretto Ghione ${ }^{1}$

Recebimento em 26/06/2017

Aceito em 07/07/2017

\section{RESUMEM}

Como ocurrió con trabajo decente, el uso de un nuevo término en el campo del Derecho del Trabajo como pasa ahora con sostenibilidad, hace que no deba soslayarse la novedad de su aparición y corresponda, por el contrario, detenerse en indagar su semántica a efectos de discernir si nos encontramos ante una invención que aporte capacidad explicativa o ante una simple "moda" intelectual que sirve para calificar casi cualquier fenómeno y por lo tanto, poco dice en lo sustantivo. El articulo trata de seguir la pista de sostenibilidad y fijar algunos significados.

Palabras Clave: Sostenibilidad; Empleos verdes; Trabajo Decente; Ambiente de trabajo; Salud y Seguridad Laboral.

\begin{abstract}
ABASTRACT
As it was the case with decent work, the use of a new term in the field of the labour law as it happens now with sustainability, makes the novelty of their appearance cannot be ignored and appropriate, by contrast, stop at investigating its semantics for the purposes of discern if we find ourselves before an invention that provides explanatory capacity or a simple intellectual "fashion" which serves to qualify nearly any phenomenon and therefore, little says in the substance. The article tries to keep track of sustainability and fix some meanings.
\end{abstract}

Keywords: Sustainability; green Jobs; decente work; work enviroment; health and safety at work.

\footnotetext{
${ }^{1}$ Profesor en Derecho de Trabajo y de Seguridad Social de la Facultad de Derecho de la Universidad de la República (Uruguay)
} 


\section{LA SOSTENIBILIDAD EN EL CAMPO JURÍDICO LABORAL. PERPLEJIDADES.}

La aparición de la locución sostenibilidad en el Derecho del Trabajo y la Seguridad Social comporta una novedad relativamente reciente si se la compara con la panoplia de términos más frecuentemente empleados en el lenguaje jurídico de nuestra disciplina. En el ámbito nacional se ha incorporado en ciertos discursos de los actores de las relaciones laborales, que toman la referencia procedente de documentos, informes, directrices, resoluciones, pareceres, etc, de los organismos internacionales; sin embargo, ha demorado todavía en desplegarse en la doctrina jurídica, pese a que un módico y rápido inventario demuestra la versatilidad y diversidad de significados que se le ha atribuido en su corta vida en aquellos espacios donde es utilizado.

Si el significado de una palabra es su uso en el lenguaje en determinados contextos, para el caso de sostenibilidad pretendemos en esta contribución reflexionar brevemente sobre esos usos de manera crítica, ya que la multiplicidad de significados tan tempranamente desarrollados y la "apropiación" que de la palabra se hace por enclaves variopintos (en el campo del derecho, que será nuestro objeto de tratamiento, pero también en el ambientalismo y ecología, la economía, la igualdad de género, etc), puede conspirar con su potencialidad para efectivamente "decir algo" por un empleo eventualmente abusivo del término ${ }^{2}$.

Nuestra hipótesis es que el uso generalizado de sostenibilidad en el derecho del trabajo le resta capacidad explicativa: para ello, repasaremos el origen del término y sus manifestaciones más visibles y recientes en documentos de la OIT y en alguna literatura

\footnotetext{
${ }^{2}$ Ferrater Mora observa la complejidad que presenta decir, como en este caso, que "el significado de una palabra es su uso en el lenguaje". Advierte al respecto que hay que cuidarse de no tomar dogmáticamente el aserto, preguntándose, por ejemplo, qué ocurre cuando se usa "abusivamente" una palabra, situación que puede "ocurrir de dos modos: o como comienzo de un nuevo uso, o como un abuso aviesamente deliberado". Agrega además que decir uso es insuficiente, ya que resulta necesario especificar de qué uso se trata, y subrayar sus diferencias. Por otra parte, advierte que "hay términos cuyo uso se aprende rápidamente; basta ver, en efecto, cómo se usan. Pero con ciertos términos no basta; hay que explicar cómo se usan. Mas explicarlo equivale a poner en claro su significado. Y aunque éste proceda de usos, queda incorporado en el léxico de una lengua y, por así decirlo, 'objetivado'". Indagaciones sobre el lenguaje. Alianza Editorial. Madrid, 1970, p. 138, 141 y 136. Decir sostenibilidad implica en este artículo, una búsqueda de los usos y sus significados y la determinación de las condiciones de su empleo (abusividad, etc) y los contextos de utilización. Naturalmente, que el tema no se agota con esta contribución.
} 
jurídica extranjera, procurando quedarnos con algunos elementos relevantes para la dogmática laboral.

No es casual que a poco de aplicar la metodología de seguir la huella de un término nuevo en derecho del trabajo, se evoque los esfuerzos similares realizados en ocasión del lanzamiento del término "trabajo decente", ocurrido en el discurso del Director General de la OIT, Sr. Juan Somavía, y en la intervención del premio Nobel de economía, Amartya Sen, en la Conferencia Internacional del Trabajo de 1999.

La revista Derecho Laboral dio rápida cuenta de la novedad ${ }^{3}$ y distintas actividades y publicaciones en nuestro medio fueron tratando de precisar, medir y comprender el fenómeno 4

Pero las analogías se quedan en la simple constancia de la novedad terminológica de ambos casos, dado que, a diferencia de sostenibilidad, que se instala en el discurso del derecho del trabajo proviniendo de otras disciplinas, trabajo decente - por el contrario - fue una invención propia del campo de lo laboral, y toda su trayectoria lo terminó entronizando como una especie de síntesis de objetivos y políticas de la Organización Internacional del Trabajo, así como un contenido frecuente y decisivo de los documentos más importantes, como el caso de la Declaración sobre la Justicia Social para una Globalización Equitativa (2008) y los programas y proyectos de cooperación con los mandantes.

Este juego de aproximaciones y distanciamientos entre sostenibilidad y trabajo decente no acaba sin embargo en la diferenciación que acabamos de hacer, por más decisiva que parezca, sino que por el contrario una serie de instrumentos internacionales recientes los vinculan muy estrechamente, desatando así un binomio sobre el que habrá que detenerse en su análisis para verificar si efectivamente nos dicen algo de perspectiva de futuro para nuestra disciplina.

En todo caso, sostenibilidad configura inevitablemente un nuevo discurso y una novedad en el lenguaje del derecho del trabajo y eso lo hace merecedor de la mayor

\footnotetext{
${ }^{3}$ Barretto Ghione, Hugo. "Concepto y dimensiones del trabajo decente: entre la protección social básica y la participación de los trabajadores en la empresa", rev. Der. Lab. T XLIV N² 204, p. 695

${ }^{4}$ Tal el caso de Oscar Ermida Uriarte, en "Concepto y medición del trabajo decente", documento preparado para el Secretariado pro témpore del Grupo Bologna/Castilla-La Mancha, Montevideo, 2000 y en el libro Trabajo Decente y Formación Profesional, OIT/CINTERFOR, 2001
} 
atención para determinar si efectivamente nos encontramos con una herramienta lingüística de interés para desarrollar rumbos y contenidos de nuestra disciplina que la actualicen frente a las demandas de los cambios, o si se trata, por el contrario, de una moda a la que son afectos muchos intelectuales, o aún una forma de gattopardismo jurídico para que la renovación del lenguaje se comporte como un simulacro de transformaciones inexistentes.

\section{ORIGEN Y PRIMER DESARROLLO DEL TÉRMINO}

Es habitual encontrar que los autores que han estudiado el origen y la procedencia del conceptos de sostenibilidad y su homónimo, desarrollo sostenible, ubiquen su raíz en el documento titulado "Nuestro futuro común", de 1987, elaborado por la Primera Ministra de Noruega, Gro Harlem Brundtland. De ese documento devino la definición clásica de desarrollo sostenible, entendido como aquel que "satisface las necesidades del presente sin comprometer la capacidad de las generaciones futuras para satisfacer sus propias necesidades". Anotemos desde la ya la importancia que reviste la "anticipación" de los efectos en las decisiones del presente, un elemento que es tenido como como principio de la sostenibilidad, y que tendrá su incidencia en lo laboral según veremos en III.4.

Los primeros pasos del término sostenibilidad lo encuentran referenciados en la preocupación en torno a las cuestiones ambientales, y la expresión hizo rápida carrera en el ámbito académico y en el de las políticas públicas, figurando en planes y proyectos vinculados a la economía, el medioambiente, el urbanismo, la salud o la educación, situando en polos dialécticos a los conceptos de crecimiento y desarrollo.

En concreto, la diferenciación corre por cuanto el crecimiento mide el bienestar y la riqueza de manera casi exclusivamente cuantitativa, teniendo como indicador paradigmático al Producto Bruto Interno (PBI) y sin que sea necesario su impacto en la mejora de la calidad de la vida humana, por lo cual desarrollo se ubica como un término definitorio de la mejora cualitativa de los recursos y servicios de que disponen las 
personas, teniendo como principal medición al Índice de Desarrollo Humano implementado por las Naciones Unidas.

Dice al respecto Fernández Buey que "Contrariamente a lo que pasa con el modelo socioeconómico dominante, que se rige por criterios de cantidad y no de calidad (producción de mercancías por medio de mercancías o producción por la producción), el simple crecimiento económico dejaría de ser el objetivo de la sostenibilidad. Es desde esta perspectiva desde la que se suele decir que desarrollo y crecimiento no son sinónimos, ya que el objetivo del desarrollo no es tener más sino vivir mejor”, por lo cual considera que debemos hablar de una "filosofía de la sostenibilidad".

Agrega: "la filosofía de la sostenibilidad tiende a matizar la noción misma de desarrollo atendiendo, por una parte, a la compatibilidad del desarrollo económico con los ecosistemas y, por otra, tomando en consideración índices de bienestar que ya no quedan reducidos a lo que sea en un momento dado el producto interior bruto. Se entiende entonces que el desarrollo al que hay que aspirar no es un desarrollo cualquiera, sino un desarrollo en equilibrio dinámico, autocentrado, racionalmente planificado $\mathrm{y}$, en la medida de lo posible, basado en la biomímesis"5

En la Cumbre Mundial de Johannesburgo de 2002 se acordó la necesidad de promover la integración de los tres componentes de la sostenibilidad (desarrollo social, el desarrollo económico y la protección del medio ambiente), elementos que han de reforzarse mutuamente.

En lo que respecta al Derecho del Trabajo, estas tres dimensiones están ínsitamente presentes, ya que como ha dicho el Director General de la OIT en su informe de 2007, "el lugar de trabajo reúne las dimensiones social, económica y medioambiental de manera indisociable"6

\section{DIMENSIONES DE LA SOSTENIBILIDAD EN EL DERECHO DEL TRABAJO}

\footnotetext{
5 Fernández Buey, Francisco. "Filosofía de la Sostenibilidad" en http://www.upf.edu/materials/polietica/_pdf/sosfilosofiasostenibilidad.pdf (consultado 29/03/2016)

${ }^{6}$ Ver Memoria "El Trabajo Decente para un Desarrollo Sostenible" para la $96^{\circ}$ Conferencia Internacional del Trabajo (2007)
} 
El discurso de la sostenibilidad se aloja en el derecho del trabajo mediante su uso progresivo en documentos e instrumentos de la Organización Internacional del Trabajo, donde aparece calificando y modulando el concepto de empresa, el ambiente de trabajo, el trabajo decente, la protección social y el empleo.

Veremos con cierto detalle cada una de esas referencias.

\subsection{EMPRESAS SOSTENIBLES Y "ENTORNO PROPICIO" PARA SU DESARROLLO}

El instrumento de mayor relevancia en cuanto a la definición de sostenibilidad y su impacto en el derecho del trabajo lo encontramos en las "Conclusiones relativas a la promoción de empresas sostenibles" alcanzada en la Conferencia Internacional del Trabajo del año 2007.

El documento reconoce que las empresas sostenibles "son una fuente principal de crecimiento, creación de riqueza, empleo y trabajo decente" y la promoción de empresas sostenibles constituye "una herramienta importante para el logro del trabajo decente, el desarrollo sostenible y la innovación que a la larga mejora los niveles de vida y las condiciones sociales", por lo cual "Los gobiernos y los interlocutores sociales tienen que cooperar para promover la integración de los tres componentes del desarrollo sostenible - económico, social y medioambiental - como pilares interdependientes y que se refuerzan mutuamente"

Nótese como a la noción de "crecimiento" diferenciada del "desarrollo" por su carácter cualitativo en el acceso a bienes y recursos, la OIT agrega el impacto de la sostenibilidad en el "empleo y trabajo decente", señas identitarias del propio organismo, dotando así al término de un contenido particular que refuerza, ciertamente, el sesgo o polo conceptual "desarrollista" por oposición al simple crecimiento material de la economía.

La OIT fundamenta el empleo de sostenibilidad reconociendo - implícitamente que no ha tenido origen en el mundo del trabajo, pero aduce que el organismo "disfruta de una posición única para contribuir al desarrollo sostenible mediante la promoción del 
trabajo decente porque es en los lugares de trabajo donde las dimensiones social, económica y medioambiental coexisten de manera indisociable".

El contenido más recurrido de las Conclusiones de 2007 y seguramente el de mayor difusión, es el concerniente a la serie de medidas propuestas como deseables para componer un "entorno propicio para las empresas sostenibles", según locución que se hizo multicitada en adelante.

La apertura hacia la implementación de un "entorno propicio" para la empresa debe compatibilizar "la búsqueda legítima de ganancias, uno de los principales motores del crecimiento económico, con la necesidad de un desarrollo que respete la dignidad humana, la sostenibilidad medioambiental y el trabajo decente" (num. 10). Pero como ocurrirá con la noción misma de sostenibilidad, el entorno propicio termina significando demasiadas cosas, como el mismo documento reconoce cuando dice que "abarca un gran abanico de factores, cuya importancia relativa puede variar en diferentes etapas del desarrollo y en contextos culturales y socioeconómicos distintos" (num. 11).

Mas adelante, parece el documento dirigirse hacia una síntesis de esos elementos, pero sin embargo recae en una enumeración demasiado extendida.

En suma, constituyen condiciones básicas y esenciales del entorno para el desarrollo de las empresas sostenibles según las Conclusiones (num. 11):

Paz y estabilidad política ${ }^{7}$.

Buena gobernanza ${ }^{8}$.

Diálogo social ${ }^{9}$.

Respeto de los derechos humanos universales y de las normas internacionales del trabajo $^{10}$.

\footnotetext{
7 “La paz y la estabilidad política son condiciones previas básicas para impulsar la constitución y el crecimiento de empresas sostenibles, mientras que la guerra y los conflictos civiles son importantes factores que desincentivan la inversión y el desarrollo del sector privado"

8 "Unas instituciones políticas democráticas, unas entidades públicas y privadas transparentes y que rindan cuentas, unas medidas eficaces de lucha contra la corrupción, y una gobernanza empresarial responsable son condiciones clave para que las economías de mercado y las empresas obtengan mejores resultados y se adecuen mejor a los valores y los objetivos a largo plazo de la sociedad"

9 “El diálogo social basado en la libertad sindical y de asociación y el derecho de negociación colectiva, en particular a través de marcos institucionales y normativos, es esencial para lograr resultados eficaces, equitativos y mutuamente beneficiosos para los gobiernos, los empleadores, los trabajadores, y la sociedad en su conjunto"

10 "La competitividad debería fundarse en valores. El respeto de los derechos humanos y de las normas internacionales del trabajo, especialmente la libertad sindical y de asociación y la negociación colectiva, la
} 
Cultura empresarial ${ }^{11}$.

6) Política macroeconómica acertada y estable y buena gestión de la economía ${ }^{12}$.

7) Comercio e integración económica sostenible ${ }^{13}$.

8) Entorno jurídico y reglamentario propicio $^{14}$.

9) Estado de derecho y garantía de los derechos de propiedad ${ }^{15}$.

10) Competencia leal ${ }^{16}$.

abolición del trabajo infantil, del trabajo forzoso y de todas las formas de discriminación, es un rasgo distintivo de las sociedades que han logrado integrar con éxito la sostenibilidad y el trabajo decente".

11 "El reconocimiento por parte de los gobiernos y la sociedad de la función clave de las empresas en el desarrollo y el firme apoyo, tanto público como privado, a la iniciativa empresarial, la innovación, la creatividad y el concepto de tutoría, sobre todo en el caso de las nuevas empresas, las pequeñas empresas, y grupos específicos tales como las mujeres y los jóvenes, son determinantes importantes de un entorno propicio para la empresa. El respeto de los derechos de los trabajadores debería incorporarse en los programas relativos a la cultura empresarial".

${ }^{12}$ Las políticas monetarias, fiscales y cambiarias deberían garantizar unas condiciones económicas estables y predecibles. Una gestión económica racional debería equilibrar los dos objetivos de crear más y mejores empleos y de combatir la inflación, y prever políticas y reglamentaciones que estimulen la inversión productiva a largo plazo. También se debería prestar atención a aumentar la demanda agregada como fuente de crecimiento económico en función de las condiciones nacionales. En el caso de los países en desarrollo y menos desarrollados, lograr unas condiciones macroeconómicas adecuadas requiere generalmente el apoyo decisivo de la comunidad internacional a través del alivio de la carga de la deuda y de la ayuda oficial para el desarrollo".

13 "Al suprimir los obstáculos que impiden el acceso a los mercados nacionales y extranjeros se deben tener en cuenta los distintos niveles de desarrollo de los países. Los aumentos de la eficiencia generados por la integración económica pueden dar lugar a efectos positivos en el empleo ya sea en términos de cantidad o de calidad o una combinación de ambos. No obstante, dado que la liberalización del comercio también puede dar lugar al desplazamiento de puestos de trabajo, una mayor informalidad y el aumento de las desigualdades en materia de ingresos, los gobiernos deben adoptar medidas, en consulta con los interlocutores sociales, para evaluar mejor la incidencia de las políticas comerciales en el empleo y el trabajo decente. También es necesario adoptar medidas a nivel regional y multilateral para eliminar las distorsiones comerciales y ayudar a los países en desarrollo a fortalecer su capacidad para exportar productos de valor añadido, gestionar el cambio y desarrollar una base industrial competitiva".

14 "Una reglamentación mal concebida y la imposición de cargas burocráticas innecesarias a las empresas limitan la creación de nuevas empresas y las actividades empresariales en curso de las empresas existentes y conducen a la informalidad, la corrupción y costos derivados de la pérdida de eficiencia. Las reglamentaciones bien concebidas, transparentes, responsables y debidamente difundidas, comprendidas las que respaldan las normas laborales y medioambientales, son buenas para los mercados y la sociedad, facilitan la formalización e impulsan la competitividad sistémica. La reforma reglamentaria y la supresión de los obstáculos a los que hacen frente las empresas no deberían menoscabar dichas normas".

15 "Un sistema jurídico formal y eficaz que garantice a todos los ciudadanos y empresas que los contratos se respetan y se cumplen, que se respeta el imperio de la ley y que se garantizan los derechos de propiedad es una condición fundamental no sólo para atraer la inversión, sino también para generar certidumbre y cultivar la confianza y la justicia en la sociedad. La propiedad es más que la mera titularidad; la ampliación de los derechos de propiedad puede ser una herramienta de habilitación y puede facilitar el acceso al crédito y el capital. Esos derechos también conllevan la obligación de cumplir las normas y los reglamentos establecidos por la sociedad“"

16 "Es necesario establecer normas sobre la competencia para el sector privado que incluyan el respeto universal de las normas laborales y sociales, y eliminar las prácticas anticompetitivas a nivel nacional" 
11) Acceso a los servicios financieros ${ }^{17}$.

12) Infraestructura material ${ }^{18}$.

13) Tecnologías de la información y la comunicación ${ }^{19}$.

14) Educación, formación y aprendizaje permanente ${ }^{20}$.

15) Justicia social e inclusión social ${ }^{21}$.

16) Protección social adecuada. ${ }^{22}$

17) Gestión responsable del medio ambiente ${ }^{23}$.

17 "Un sistema financiero que funciona bien es el catalizador del crecimiento de un sector privado dinámico. Si se facilita el acceso de las PYME, con inclusión de las cooperativas y las empresas incipientes, a la financiación, por ejemplo, al crédito, el arrendamiento financiero, los fondos de capital de riesgo u otros tipos de instrumentos similares o nuevos, se crean las condiciones apropiadas para un proceso más incluyente de desarrollo de las empresas. Habría que alentar a las instituciones financieras, particularmente las multilaterales e internacionales, a incluir el trabajo decente en sus prácticas crediticias”.

18 "La sostenibilidad de las empresas y el desarrollo humano dependen fundamentalmente de la calidad y cantidad de la infraestructura existente, como la infraestructura material para las empresas, los sistemas de transporte, las escuelas y los hospitales. El acceso seguro y asequible al agua y la energía también constituye un gran desafío, especialmente en los países en desarrollo. Ayuda también particularmente a las empresas el acceso local a sectores de actividad auxiliares como los proveedores de servicios y los proveedores y fabricantes de maquinaria".

19 “Ampliar el acceso a las tecnologías de la información y la comunicación (TIC) es otro desafío fundamental en la era de la economía del conocimiento. El uso de las TIC es, pues, fundamental para el desarrollo de empresas sostenibles, por lo que debe ser plenamente utilizado a dicho fin. La tecnología de banda ancha asequible es también de suma importancia para los países y empresas, y debería facilitarse".

20 “El talento humano es el factor productivo más importante de la economía contemporánea. Es importante centrarse en el desarrollo de una fuerza de trabajo calificada y el aumento de las capacidades humanas a través de sistemas de educación, formación y aprendizaje permanente de alta calidad para ayudar a los trabajadores a encontrar buenos empleos y a las empresas a encontrar los trabajadores calificados que necesitan. Asimismo, se debería proporcionar apoyo financiero para mejorar el acceso de los trabajadores pobres a la formación y el perfeccionamiento de las calificaciones. De ese modo, la sociedad podrá alcanzar el doble objetivo del éxito económico y el progreso social".

21 "La desigualdad y la discriminación son incompatibles con el desarrollo de empresas sostenibles. Se requieren políticas claramente definidas con miras a la justicia social, la inclusión social y la igualdad de oportunidades en el empleo. El ejercicio efectivo del derecho a organizarse y a la negociación colectiva también constituye un medio eficaz para garantizar la distribución equitativa de los aumentos de productividad y la remuneración adecuada de los trabajadores".

22 "Un modelo de seguridad social universal sostenible basado en los impuestos o cualquier otro modelo nacional que proporcione a los ciudadanos acceso a servicios esenciales tales como una atención de salud de calidad, prestaciones de desempleo, la protección de la maternidad y una pensión básica, es fundamental para mejorar la productividad y propiciar las transiciones a la economía formal. La protección de la salud y la seguridad de los trabajadores en el lugar de trabajo también es vital para el desarrollo de empresas sostenibles".

23 "En ausencia de reglamentaciones e incentivos adecuados, los mercados pueden dar lugar a resultados no deseados para el medio ambiente. Se deberían utilizar los incentivos y reglamentaciones fiscales, incluidos los procedimientos de contratación pública, para promover pautas de consumo y producción que sean compatibles con las exigencias del desarrollo sostenible. Las soluciones basadas en los mercados privados, como el empleo de criterios medioambientales al evaluar el riesgo de crédito o el rendimiento de la inversión, constituyen igualmente medios eficaces para hacer frente a ese problema". 
Como puede verse, la extensión desmesurada del "entorno propicio" hace que comprenda prácticamente la totalidad de los componentes de las políticas económicas, tributarias, laborales, previsionales, etc, incluyendo además elementos propios de una declaración de derechos de los trabajadores y no de un conjunto de medidas de índole política dirigidas a facilitar el desenvolvimiento empresarial. Obviamente esa conjunción de elementos variopintos (derechos de los trabajadores/medidas de desarrollo empresarial) responden a una especie de transacción de valores entre los actores, pero termina configurando como resultado de ese acuerdo un concepto de empresa sostenible que incorpora componentes éticos, instrumentales y de derechos.

Por otra parte, alguno de los elementos integrantes del "entorno propicio" aparecen reiterados en el documento cuando se enumeran los "principios" de las empresas sostenibles (num. 13):

1) Diálogo social y buenas relaciones laborales: "Las empresas sostenibles participan en el diálogo social y buenas relaciones laborales como la negociación colectiva y la información, consulta y participación de los trabajadores. Estos son instrumentos eficaces para crear situaciones en las que todas las partes salgan ganando, ya que promueven valores comunes, la confianza y cooperación, y un comportamiento socialmente responsable"

2) Desarrollo de los recursos humanos: "El desarrollo de los recursos humanos en las empresas sostenibles debería basarse en el diálogo social y la participación de los trabajadores".

3) Condiciones de trabajo: "Las empresas sostenibles ofrecen condiciones de trabajo que proporcionan un entorno de trabajo seguro y motivador y una organización del trabajo flexible y mutuamente beneficiosa". El numeral abunda luego en el respeto a los derechos fundamentales del trabajador emergentes de la Declaración sobre los Principios y Derechos Fundamentales en el trabajo de 1998.

En un curioso intento de armonizar intereses diversos, el documento de Conclusiones dice, luego de reconocer que el Repertorio de recomendaciones prácticas de la OIT sobre el VIH/SIDA y el mundo del trabajo es una referencia útil, que "Las empresas sostenibles reconocen que tales prácticas mejoran la productividad y refuerzan la capacidad de innovación y la competitividad de la empresa”. 
La expresión solo puede entenderse en el contexto del debate frente a los partidarios de la promoción de la competitividad empresarial con base a la baja de condiciones de trabajo, ya que de lo contrario no se explica que se pretenda una tan directa vinculación entre los derechos de las personas y la mejora de la productividad y la competitividad.

4) Productividad, salarios y beneficios compartidos: "Los trabajadores tienen que poder participar en el éxito de las empresas y obtener una parte justa de los beneficios derivados de las actividades económicas y del aumento de la productividad"

$5 \mathrm{La}$ responsabilidad social de la empresa (RSE): "La RSE no puede sustituir a la normativa jurídica ni a la aplicación de la ley ni a la negociación colectiva. No obstante, cuando la RSE es transparente y creíble y está basada en una genuina asociación, puede proporcionar a los trabajadores y otras partes interesadas más oportunidades para lograr que las empresas se comprometan con respecto a las repercusiones sociales y medioambientales de sus actividades".

6) Gobernanza empresarial y prácticas empresariales.

El documento de Conclusiones agrega que "Los principios de las empresas sostenibles deberían promoverse a lo largo de las cadenas de suministro".

Este último término, "cadenas de suministro", está haciendo camino en la OIT como derivación del concepto de empresa en el actual contexto productivo mundial, y desde ya debemos dejar señalada la importancia de no desatender su evolución en los próximos años.

En definitiva, la reiteración de los elementos que constituyen los objetivos políticos y éticos del "entorno propicio" (num. 11) para el desarrollo de las empresas por una parte, y de los derechos que componen los principios que deben conducir la conducta de las empresas sostenibles (num. 13) por otra, denota que más que la precisión y el discernimiento conceptual entre políticas y derechos, prevaleció un intento transaccional de puntos de vista distintos que no aporta claridad en la conceptuación de que es, en definitiva, una empresa sostenible, ya que no puede ser todo a la vez o al menos deberían privilegiarse algunos elementos centrales que distan de apreciarse, confundidos como están como en un palimpsesto. 


\section{III.2 PAPEL DE LA SOSTENIBILIDAD EN EL TRÁNSITO AL TRABAJO FORMAL}

Vinculado al mundo de la empresa, el término aparece referido en la reciente Recomendación num 204 sobre la Transición de la Economía Informal a la Economía Formal, de 2015.

El cap. III sobre los marcos jurídicos y de políticas establece que "Los Miembros deberían llevar a cabo una evaluación y un diagnóstico adecuados de los factores, características, causas y circunstancias de la actividad informal en el contexto de cada país, a fin de fundamentar adecuadamente la formulación y la aplicación de leyes, políticas y otras medidas destinadas a facilitar la transición a la economía formal”, y en ese marco, debería abordarse "la promoción de estrategias de desarrollo sostenible, erradicación de la pobreza y crecimiento inclusivo, y la generación de empleos decentes en la economía formal" (num. 11).

En relación al fomento del empleo, las consultas tripartitas que se implementen podrían incluir las referidas a las "políticas macroeconómicas (...) que respalden la demanda agregada, la inversión productiva y la transformación estructural, promuevan empresas sostenibles, sustenten la confianza de las empresas y corrijan las desigualdades" y las "políticas empresariales que fomenten empresas sostenibles y que favorezcan en particular las condiciones para un entorno propicio (...) lo que comprende el apoyo a las microempresas, a las pequeñas y medianas empresas, al emprendimiento y a normativas bien diseñadas, transparentes y adecuadamente difundidas que faciliten la transición a la economía formal y una competencia leal” (num. 15).

Aquí aparece una utilización del "entorno propicio" para la creación de empresas sostenibles como política tendiente a apoyar el desarrollo de micro, pequeñas y medianas empresas, medidas todas tendientes a facilitar la transición a la economía formal. Sostenibilidad se trasunta en instrumento de política social.

\subsection{SOSTENIBILIDAD, “EMPLEOS VERDES” Y TRABAJO DECENTE}


La literatura reciente de algunos organismos internacionales, en particular la OIT, ha visto emerger otra novedad del lenguaje, esta vez a través de la noción de "empleos verdes", cuestión muy vinculada, como se verá, al desarrollo sostenible y a las empresas sostenibles.

Se trata de una síntesis entre las distintas dimensiones de Sostenibilidad en su vertiente ambientalista y en su concepción económica cualitativa (frente a la más clásica de crecimiento cuantitativo desinteresado de las cuestiones de desarrollo) y el empleo, calificado ahora a partir de una perspectiva de tipo alternativo, sumando así un nuevo componente a la noción de trabajo decente.

En suma, sostenibilidad, empleos verdes y trabajo decente se interrogan e integran en una construcción lingüística formulada a partir de procedencias del campo de la economía, el medioambiente, el empleo y los derechos de las personas que trabajan.

En la Resolución de la OIT sobre el Desarrollo Sostenible, el Trabajo Decente y los Empleos Verdes (2013) se define el primero de los términos ${ }^{24}$ y se dice luego con total transparencia que "Los cuatro pilares del Programa de Trabajo Decente - diálogo social, protección social, derechos en el trabajo y empleo - son componentes indispensables del desarrollo sostenible que deben ponerse en el centro de las políticas encaminadas a lograr un crecimiento y un desarrollo sólidos, sostenibles e incluyentes".

Con esto queda dicho que la sostenibilidad se basa en el trabajo decente y que éste, a su vez, debe ponerse en el centro de las políticas de desarrollo sostenible, con lo cual el discurso parece bastante circular o tautológico (sostenibilidad/trabajo decente/desarrollo sostenible).

Con independencia de esta omnipresencia de la sostenibilidad en el inicio y el final de la formulación del texto, lo relevante es que proyecta al trabajo decente introduciendo así los derechos fundamentales y el diálogo social en la noción misma de sostenibilidad laboral.

\footnotetext{
${ }^{24}$ En concreto, se indica que "Se entiende por desarrollo sostenible aquel que permite satisfacer las necesidades de la generación actual sin restar capacidad a las generaciones futuras para satisfacer las suyas. El desarrollo sostenible abarca tres dimensiones - la económica, la social y la ambiental — que están interrelacionadas, revisten igual importancia y deben abordarse conjuntamente”.
} 
La Resolución señala las vias de tránsito hacia "economías y sociedades ambientalmente sostenibles" (num. 13) debería regirse por principios tales como "un consenso social sólido sobre el objetivo de la sostenibilidad y las vías para alcanzarlo" (lit. a) y la promoción de "la creación de más empleos decentes e incluso, si procede, prever la repercusión en el empleo y favorecer una protección social adecuada $\mathrm{y}$ sostenible frente a la pérdida de empleos y los desplazamientos, así como el desarrollo de competencias y el diálogo social, incluido el ejercicio efectivo del derecho de sindicación y de negociación colectiva" (lit. e).

Ingresando al cerno mismo de la cuestión de los componentes ecológicos de esa transformación de la economía y las empresas, la Resolución expresa que son elementos de un marco básico "La ecologización de la economía en el contexto del desarrollo sostenible y la erradicación de la pobreza" (num. 14) que "hará necesaria una combinación de políticas macroeconómicas, industriales, sectoriales y laborales específicas para cada país que creen un entorno propicio para que prosperen las empresas sostenibles y generen oportunidades de trabajo decente movilizando y dirigiendo la inversión pública y privada hacia actividades ambientalmente sostenibles (...) El objetivo debería ser la creación de trabajo decente en toda la cadena de suministro, en sectores dinámicos de gran valor añadido que fomenten la mejora del empleo y las competencias profesionales, así como la creación de empleo y una mayor productividad en industrias más intensivas en mano de obra que ofrezcan oportunidades de empleo a gran escala".

El papel de los gobiernos es fundamental: al respecto, dice que la Resolución que “deberían fomentar la ecologización de los empleos mediante marcos normativos y no normativos que apoyen la sostenibilidad ambiental y social, y al mismo tiempo estimulen la innovación y las inversiones tanto en el propio país como en el extranjero".

En cuanto al desarrollo de competencias, se señala que "Debe haber una interacción sólida entre el mundo del trabajo y el de la educación y la formación. A fin de tener éxito, la ecologización de la economía debe centrarse en el desarrollo de competencias. Unos sistemas de educación y formación técnica y profesional sólidos deben incluir la participación de la industria y los sindicatos” (lit. g). 
Sobre el vital punto de la seguridad y salud en el trabajo se indica que se debe “Garantizar que todos los empleos, incluidos los empleos verdes, sean decentes, seguros y saludables es un aspecto fundamental de la mejora de la calidad del empleo. Las normas y la formación en materia de seguridad y salud en el trabajo deben ser un componente esencial de todas las actividades de desarrollo de las competencias profesionales. Deberían adoptarse medidas prácticas de prevención en las empresas sobre la base de la evaluación del riesgo y los principios de la eliminación y el control de peligros" (lit. i)

Los sistema de Protección social también deben ser sostenibles para esta Resolución sobre Empleos Verdes, Trabajo Decente y Sostenibilidad: “Los regímenes de protección social sólidos, integrales y sostenibles constituyen una parte integrante de la estrategia para la transición hacia un modelo de desarrollo sostenible basado en los principios del trabajo decente, la justicia social y la inclusión social. Esos regímenes deberían proporcionar a los trabajadores que han quedado desplazados debido al cambio tecnológico, o a los afectados por desastres naturales, apoyo en materia de ingresos, así como acceso a la atención de salud y a servicios básicos durante la transición, y de ese modo reducir las desigualdades" (lit. j).

En el plano de los derechos se subrayan algunos muy relevantes, en tanto no solamente se requiere de aquellos que integran los considerados como fundamentales desde la Declaración de 1998, sino que se incluyen otros que hacen parte de los convenios prioritarios (como consultas tripartitas e inspección del trabajo) o aún los no incluidos de manera expresa en esos elencos privilegiados: "Las normas internacionales del trabajo ofrecen un marco sólido para hacer frente a los desafíos del mundo del trabajo relacionados con la ecologización de la economía y, de manera más general, con la transición hacia el desarrollo sostenible y la erradicación de la pobreza. Algunas normas internacionales del trabajo sobre la libertad sindical y de asociación, entre ellas las relativas a la libertad de asociación y al derecho a la negociación colectiva, la prohibición del trabajo forzoso, el trabajo infantil y la no discriminación, el diálogo social, las consultas tripartitas, el salario mínimo, la administración e inspección del trabajo, las políticas de empleo, el desarrollo de los recursos humanos, la seguridad y salud en el trabajo, y la seguridad social, son importantes a este respecto" (lit. m). 
El vínculo sostenibilidad y trabajo decente ha tenido otras recientes manifestaciones de interés por los compromisos internacionales que denotan ${ }^{25}$.

Fuera de cierta voluntariedad que se aprecia en lo elevado de los propósitos, lo sustantivo para esta oportunidad es verificar la alta consideración que merece el trabajo decente dentro de los objetivos de la comunidad internacional.

\subsection{SOSTENIBILIDAD Y SALUD Y SEGURIDAD EN EL TRABAJO.}

Según se ha visto, la sostenibilidad en su origen se relaciona con conceptos ambientales como precaución y anticipación de los cambios y evitabilidad de los daños o perjuicios futuros.

En el derecho del trabajo, la noción de "anticipación" como tal no tiene un lugar todavía muy asentado, aunque se la ha visto materializada en institutos tales como los despidos por razones económicas y en el derecho a la salud y seguridad en el trabajo ${ }^{26}$. En este último sentido, la anticipación "ya no se entiende sólo como la exigencia de un comportamiento diligente propio del buen padre; la anticipación implica que el empleador es responsable de los riesgos generados por su actividad"27. Esta verdadera obligación de resultado, si bien es clásica en el derecho del trabajo y la seguridad social, tiene en sostenibilidad un argumento adicional para su fundamento.

La "anticipación" como principio de la sostenibilidad no se traduce en la sola previsión del daño, noción totalmente dependiente de la probabilidad de conocimiento de la concurrencia del riesgo para reducir el perjuicio, sino que se traduce en el concepto de

\footnotetext{
${ }^{25}$ En las "Directrices de Politica para una transición justa hacia economías y sociedades ambientalmente sostenibles para todos", adoptada en la Conferencia de 2015, se dio seguimiento a la Recomendación glosada supra a través de una reunión de expertos. Finalmente, en la Agenda 2030 para el Desarrolllo Sostenible de las Naciones Unidas se fijan 17 objetivos, uno de los cuales es "Promover el crecimiento económico sostenido, inclusivo y sostenible, el pleno empleo productivo y el trabajo decente para todos, lo que constituye una nueva secuela de la relación sostenibilidad/trabajo decente. Entre las metas de la Agenda figura para el año 2030 lograr el empleo pleno y productivo y garantizar un trabajo decente para todos y la igualdad de remuneración por trabajo de igual valor; para 2020, entre otros objetivos, "proteger los derechos laborales y promover un entorno de trabajo seguro y protegido para todos los trabajadores, incluidos los trabajadores migrantes, en particular las mujeres migrantes y las personas con empleos precarios".

${ }^{26}$ Alzais, Sabrina. "El Derecho del Trabajo frente a los nuevos retos del desarrollo sostenible" en el vol. La Ecología del Trabajo. Laura Mora Cabello del Alba y Juan Escribano Gutiérrez ed. Bomarzo, 2015, p. 159

${ }^{27}$ Alzais, ob cit. p. 167
} 
prevención, demandando medidas positivas para conjurar el riesgo, siempre que tengan la capacidad para garantizar la seguridad de los trabajadores. Esta es la vía por la cual la anticipación, como principio de realización del desarrollo sostenible, se extiende al Derecho del Trabajo ${ }^{28}$.

El encuentro entre Derecho del Trabajo y Ambiente Laboral provoca que la normativa laboral se tiña fuertemente de contenidos de salud y seguridad y asuma en consecuencia cada vez mayor cantidad de riesgos objeto de prevención, ampliando la esfera de derechos objeto de protección. El bien jurídico pasa a ser la integridad psicofísica de la personas y/o su dignidad. En este sentido, el Convenio Internacional del Trabajo núm. 155 aportó un decisivo avance desde la misma formulación de su título (sobre seguridad y salud de los trabajadores y medio ambiente de trabajo) atando así la seguridad y salud a las cuestiones ambientales en el trabajo.

\section{CONCLUSIONES}

Al final del trayecto, parece constatarse que efectivamente el término sostenibilidad tiene una gama amplia de significados producto de diversos usos aún en el ámbito del Derecho del Trabajo, demostrativo de su ductilidad para abarcar las áreas de la economía, la sociedad y el medioambiente.

La consideración de los problemas ecológicos en el trabajo reenvía a la cuestión del modelo productivo y sus impactos en el ambiente: así, el conflicto ambiental llega tardíamente a anidar en un escenario surcado por el conflicto económico como es el laboral, por lo cual su recepción no se da sin perplejidades y contradicciones. Es posible, sin embargo, compatibilizar el desarrollo sostenible con el empleo, a condición que la discusión se desplace hacia un cuestionamiento del modelo de producción existente ${ }^{29}$.

\footnotetext{
${ }^{28}$ Alzais, ob cit. p. 173

${ }^{29}$ En efecto, en principio podría sostenerse que los trabajadores necesitan que el modelo productivo funcione a pleno para asegurar los niveles de empleo, por lo cual toda medida ambiental puede ser vista a contrapelo de estas demandas del crecimiento económico. Sin embargo, los empleos verdes han comenzado a emerger como alternativa, compatibilizando el mundo del trabajo con las preocupaciones ambientales:
} 
El concepto de empresa sostenible denota alguna ambigüedad a partir de la reiteración de los elementos constitutivos del "entorno propicio" para su creación, y de los principios que deben conducir la conducta de las empresas sostenibles, incluyendo derechos de los trabajadores y resguardos ambientales, etc. Seguramente obró en esta instancia un intento transaccional de puntos de vista distintos de los actores, pero el resultado final es un tanto recargado y repetitivo, como si se encontraran sobreimpresos en un palimpsesto.

La sostenibilidad se basa en el trabajo decente y éste, a su vez, debe ponerse en el centro de las políticas de desarrollo sostenible, con lo cual el discurso parece tautológico (sostenibilidad/trabajo decente/desarrollo sostenible). Con independencia de esta omnipresencia de la sostenibilidad en el inicio y el final de la formulación del texto, lo que puede rescatarse es que proyecta al trabajo decente hacia los confines de la empresa, introduciendo así los derechos fundamentales y el diálogo social en la noción misma de empresa sostenible.

En el plano de los derechos, la sostenibilidad y los empleos verdes deben cumplir no solamente con los Derechos Fundamentales de la Declaración de la OIT de 1998, sino que en algunos documentos del organismo se incluyen otros derechos, que hacen parte de los convenios prioritarios (como consultas tripartitas e inspección del trabajo) o aún no fueron incluidos de manera expresa en esos elencos privilegiados, como el caso del salario mínimo.

Algunos autores han llevado el objetivo de la sostenibilidad al centro mismo del juego de obligaciones y derechos en la relación de trabajo. Así, Perez Amorós señala que en el mundo del trabajo actual "existen dos objetivos que cada vez adquieren mayor protagonismo: que el trabajo a prestar por los asalariados sea "justo" y "digno"; y que el desarrollo socio-económico al que se aspira con la organización y prestación de dicho trabajo sea "sostenible", entendiendo por tal la búsqueda del progreso económico siempre necesario- sin menosprecio alguno a la calidad de vida ni deterioro del medioambiente. Ambas exigencias, son, a la vez, razones generales que explican la

ver al respeto el número monográfico del Boletín Internacional de Investigación Sindical, OIT 2012, vol. 4, núm. 2, que destaca la importancia de la producción con protección ambiental en el sentido de demandar más trabajo humano que el productivismo capitalista. 
conexión existente entre el Derecho que profesamos y la ordenación del medio ambiente; o dicho en otras palabras que nos permitan avanzar más en nuestro planteamiento: la integración del medio ambiente como contenido esencial del trabajo"30.

Estas aportaciones de sostenibilidad al Derecho del Trabajo no evitan que aparezca a menudo referida de manera abusiva o que en muchos casos termine designando de distinta forma institutos tradicionales del derecho del trabajo (ejemplo claro de ello parece ser el caso de la "anticipación" en materia de salud y seguridad). No obstante, también es posible postular que en algunas hipótesis esta reiteración de significados puede contribuir a fundar más sólida y “modernamente” los derechos laborales.

En síntesis, y pese a esos problemas del uso del término sostenibilidad, lo cierto es que al circular tan fluidamente en el discurso actual del derecho del trabajo, presenta una función "intertextual” cuyas manifestaciones más promisorias podrían inventariarse provisionalmente como: 1) vincula y radica los derechos fundamentales del trabajador en la noción misma de empresa sostenible; 2) enriquece la perspectiva del trabajo decente desde la vertiente ambiental; 3) denota la importancia de los aspectos ambientales y de salud laboral, reforzando compromisos y responsabilidades de tipo objetivo bajo la obligación de anticipar el riesgo (prevención) como principios del desarrollo sostenible; y por último, 4) hace de la temática ambiental un contenido esencial e indisociable de la prestación en la relación de trabajo y por ende se inserta en la trama obligacional (con insospechadas derivaciones en el plano de la ejecución del contrato y los avatares del mismo en cuanto a los eventuales incumplimientos que se produzcan por introducción de variaciones o alteraciones por parte del empleador que puedan considerarse "contra ambientales").

\footnotetext{
${ }^{30}$ Pérez Amorós. Francisco. "Derecho del trabajo y medioambiente: unas notas introductorias. En rev. Gaceta Laboral vol. 16, núm. 1, Univ. de Zulia, enero - abril 2010, p. 93
} 